\title{
New bivariate gamma types with MIMO application
}

\author{
A. Bekker ${ }^{a}$, M. Arashi ${ }^{a, b}$, and J.T. Ferreire ${ }^{1}{ }^{a}$ \\ ${ }^{a}$ Department of Statistics, University of Pretoria, Pretoria, South Africa \\ ${ }^{b}$ Department of Statistics, School of Mathematical Sciences, Shahrood University of Technology, Shahrood, Iran
}

\section{ABSTRACT}

In this paper a bivariate gamma type distribution emanating from the diagonal elements of an inverse Wishart type distribution is developed; which in turn originates from the complex matrix variate elliptical class. From this, a bivariate Weibullised gamma type distribution is also presented, of which the bivariate Nakagami- $m$ type is a special case. The derived results may be applied as decision statistics for a MIMO (multiple input multiple output) system with two transmit antennas. It is proposed that under this elliptical umbrella some performance measures such as the outage probability of MIMO systems can be analyzed in broad generality.

Keywords and phrases: Complex inverse Wishart, Complex $t$, Elliptical, Nakagami- $m$, Outage probability 2000 MSC: 62E15, 60E05, 94A99

\section{Introduction}

In the field of multiple input multiple output (MIMO) systems and wireless communications, fading channels are characterized as statistical distributions used to model and describe the signal degradation from the transmitter to the receiver of wireless signals. Certain assumptions such as geographical area, type of transmitters or receivers etc. give rise to certain distributions being preferred to describe the fading of signals. Some of these include the Rayleigh and Rician (see Miller (1974)), Hoyt (see Hoyt (1947)), and Nakagami (see Nakagami (1960)) fading. Rayleigh fading is of particular interest in this paper. de Souza and Yacoub (2008) mentioned that the Rayleigh probability density function (pdf), moreover specifically the basis for assuming Rayleigh fading in wireless communications, is usually derived based on the assumption that from the central limit theorem for large number of partial waves, the resultant process can be decomposed into two orthogonal zero mean and equal standard deviation normal random processes. This is an approximation and the restriction of complex normal is unnecessary - it is not always a large number of interfering signals. Thus a more general assumption than normal may not be that far from reality (see also Ollila et. al. (2011)).

In this paper, the complex matrix variate normal assumption in the construction and motivation of the Rayleigh fading model is relaxed and generalized to that of the complex matrix variate elliptical class. By doing so, different members of the complex matrix variate elliptical class can be assumed as underlying models and compared to that of the usual complex matrix variate normal. In particular, the complex matrix variate $t$ distribution is assumed and investigated comparatively with regards to said normal assumption. However, the complex elliptical class is assumed first in the fading environment, and subsequently investigated for the complex matrix variate $t$ case.

The complex matrix variate elliptical class is shortly reviewed next. Let $\mathbf{H}: m \times p$ be a complex matrix variate that is distributed according to the complex matrix variate elliptical distribution, denoted as $\mathbf{H} \sim C E_{m, p}\left(\mathbf{0}, \mathbf{I}_{m} \otimes \boldsymbol{\Sigma}, h\right)$, with the following pd $\mathbf{f}^{2}$

$$
f_{\mathbf{H}}(\mathbf{H})=|\boldsymbol{\Sigma}|^{-m} h\left(-\operatorname{tr} \mathbf{H} \boldsymbol{\Sigma}^{-1} \mathbf{H}^{H}\right),
$$

\footnotetext{
${ }^{1}$ Corresponding author: johan.ferreira@up.ac.za

${ }^{2} H$ denotes Hermitian transpose.
} 
with covariance matrix ${ }^{3} \boldsymbol{\Sigma}>\mathbf{0}: p \times p$ and density generator $h: \mathbb{R}^{+} \rightarrow \mathbb{R}^{+}$. Provost and Cheong (2002) reports that the pdf of this complex elliptical random matrix $\mathbf{H}$ can be represented as

$$
f_{\mathbf{H}}(\mathbf{H})=\int_{\mathbb{R}^{+}} f_{C N_{m, p}\left(\mathbf{0}, \mathbf{I}_{m} \otimes t^{-1} \mathbf{\Sigma}\right)}(\mathbf{H} \mid t) S(t) d t
$$

where $f_{C N_{m, p}\left(\mathbf{0}, \mathbf{I}_{m} \otimes t^{-1} \boldsymbol{\Sigma}\right)}(\mathbf{H} \mid t)$ is the pdf of a complex matrix variate normal distribution, denoted as $C N_{m, p}\left(\mathbf{0}, \mathbf{I}_{m} \otimes t^{-1} \boldsymbol{\Sigma}\right)$ and $S(t)$ is a weight function depending only on $t$. Note that $S(t)$ is not necessarily positive on all its domain. From representation (2) it is evident that any characteristic of complex matrix elliptical variates can be studied under a complex matrix variate normal assumption with a scale change in covariance matrix.

Next, the platform is set for matrix variates within the wireless communications framework relevant in this paper. A single user MIMO system depends on the channel propagation matrix $\mathbf{H}$ in the following manner:

$$
\mathbf{y}=\mathbf{H x}+\mathbf{v}
$$

where $\mathbf{y}, \mathbf{v} \in \mathbb{C}^{m \times 1}, \mathbf{x} \in \mathbb{C}^{p \times 1}$ and $\mathbf{H} \in \mathbb{C}^{m \times p}\left(\mathbb{C}^{m \times p}\right.$ denotes the space of complex positive definite matrices of dimension $m \times p)$. For a Rayleigh fading model, the distribution of an $m \times p$ channel matrix $\mathbf{H}$ is usually assumed to be $C N_{m, p}\left(\mathbf{0}, \mathbf{I}_{m} \otimes \boldsymbol{\Sigma}\right.$ ) distributed, with $m \geq p$ (see Miller (1974)). However, assuming a Rayleigh type fading model implies that the channel matrix $\mathbf{H}$ and noise vector $\mathbf{v}$ are independently distributed according the complex matrix variate elliptical distribution, in other words, $\mathbf{H} \sim C E_{m, p}\left(\mathbf{0}, \mathbf{I}_{m} \otimes \boldsymbol{\Sigma}, h\right)$, and (independently) $\mathbf{v} \sim C E_{m, 1}\left(\mathbf{0}, \sigma^{2} \mathbf{I}_{m}\right.$ ) (see Telatar (1999)). Thus in this paper the complex matrix variate normal assumption that $\mathbf{H}$ usually follows is substituted with the complex matrix variate elliptical distribution following the assumption of Rayleigh type fading. The following proposition presents the Rayleigh type fading under consideration in this paper.

Proposition 1 Let $Z=X+j Y$ where $j=\sqrt{-1}$, and where $X, Y$ are independent and identically zero mean elliptical random variates with common variance $\sigma^{2}$ (that is, $C E\left(0, \sigma^{2}, h\right)$ ). Let $z=\sqrt{x^{2}+y^{2}}=$ $r e^{i \theta}$ denote an element $h_{i j}$ of $\mathbf{H}$. The joint pdf of $r$ (amplitude of the fading signal) and $\theta$ (phase of the fading signal) in the complex elliptical class is given by

$$
f\left(r, \theta \mid \sigma^{2}\right)=\int_{\mathbb{R}^{+}} \frac{r}{2 \pi \sigma^{2} t^{-1}} \exp \left(-\frac{r^{2}}{t^{-1} \sigma^{2}}\right) S(t) d t
$$

with

$$
h\left(r \mid \sigma^{2}\right)=\int_{\mathbb{R}^{+}} \frac{r}{\sigma^{2} t^{-1}} \exp \left(-\frac{r^{2}}{2 \sigma^{2} t^{-1}}\right) S(t) d t,
$$

where $r>0$, as the Rayleigh type pdf. The distribution of the phase $\theta$ remains uniform, with pdf (see Miller (1974))

$$
k\left(\theta \mid \sigma^{2}\right)=\frac{1}{2 \pi}, \quad 0 \leq \theta \leq 2 \pi
$$

and zero otherwise.

In this paper the main contribution is to propose a Rayleigh type fading distribution (see Proposition 1), and subsequently derive the joint distribution of the diagonal elements of $\left(\mathbf{H}^{H} \mathbf{H}\right)^{-1}$, where $\mathbf{H} \sim$ $C E_{m, 2}\left(\mathbf{0}, \mathbf{I}_{m} \otimes \boldsymbol{\Sigma}, h\right)$; this is called a bivariate gamma type distribution and is discussed in Section 2 . In

\footnotetext{
${ }^{3}$ For a matrix $\mathbf{X}, \mathbf{X}>\mathbf{0}$ indicates the matrix is positive definite.

${ }^{4} \mathbb{R}^{+}$denotes the positive real line.
} 
addition, particular members of the complex elliptical class is investigated (specifically the complex matrix variate normal- and complex matrix variate $t$ distributions) as underlying models for the newly proposed bivariate gamma type distribution. Subsequently, a bivariate Weibullised gamma type distribution emanating from the proposed bivariate gamma type distribution is introduced, where a special case of the underlying complex matrix variate normal is a particular new contribution not studied by $\mathrm{Xu}$ et. al. (2009), in addition to the underlying complex matrix variate $t$ case. Bivariate distributions have been extensively explored in the literature; we refer to Balakrishnan and Lai (2002), de Souza and Yacoub (2008), de Souza et. al. (2012), Chen et. al. (2014), Ermelova and Tirkonnen (2015), and Mahdavi et. al. (2017) to mention some. Finally in Section 3, possible application of the derived results are proposed with regards to the outage probability and the equal gain diversity of an $m \times 2$ MIMO system; which is analyzed in a broad generality from an elliptical viewpoint, and comparatively investigated for the underlying complex matrix variate normal- and $t$ cases. Section 4 contains final conclusions.

\section{Bivariate distributions}

In this section, a new bivariate gamma type distribution, extending the work of Xu et. al. (2009), emanating from the complex matrix variate elliptical class is proposed. In particular they considered the joint distribution of the diagonal elements of $\mathbf{W}=\left[\begin{array}{ll}w_{11} & w_{12} ; w_{12}^{*} w_{22}\end{array}\right]$, where $\mathbf{W}=\left(\mathbf{H}^{H} \mathbf{H}\right)^{-1}$, with $\mathbf{H}$ : $(m \times 2)$ distributed according to a complex matrix variate normal distribution $C N_{m, 2}\left(\mathbf{0}, \mathbf{I}_{m} \otimes \boldsymbol{\Sigma}\right)$ with $\left.\right|^{5}$ $\boldsymbol{\Sigma}=\left[1 \xi ; \xi^{*} 1\right]$ as the covariance matrix. Suppose $\mathbf{H} \sim C E_{m, 2}\left(\mathbf{0}, \mathbf{I}_{m} \otimes \boldsymbol{\Sigma}, h\right)$. Then $\mathbf{S}=\mathbf{H}^{H} \mathbf{H}$ is complex matrix variate Wishart type distributed, and the pdf is given by

$$
f_{\mathbf{S}}(\mathbf{S})=\frac{|\mathbf{S}|^{m-2} \mathcal{G}(\mathbf{S})}{\Gamma_{2}(m)|\mathbf{\Sigma}|^{m}}, \mathbf{S}>\mathbf{0}
$$

where $\Gamma_{2}(m)$ denotes the complex multivariate gamma function $\bigsqcup^{6}$ and where $£^{7}$

$$
\mathcal{G}(\mathbf{S})=\int_{\mathbb{R}^{+}} t^{2 m} \operatorname{etr}\left(-t \mathbf{\Sigma}^{-1} \mathbf{S}\right) S(t) d t .
$$

\subsection{Bivariate gamma type distribution}

The following lemma defines a complex matrix variate inverse Wishart type distribution. Subsequently, a bivariate gamma type emanating from the diagonals of this complex matrix variate inverse Wishart type distribution is derived.

Lemma 2 Suppose $\mathbf{S}$ follows a complex matrix variate Wishart type distribution with pdf (3). Then $\mathbf{W}=\mathbf{S}^{-1}$ follows a complex matrix variate inverse Wishart type distribution with $p d f$

$$
\begin{aligned}
f(\mathbf{W})= & \int_{\mathbb{R}^{+}} \frac{t^{2 m}}{\Gamma_{2}(m)}\left(\frac{1}{w_{11} w_{22}-w_{12} w_{12}^{*}}\right)^{m+2}\left(\frac{1}{1-\xi \xi^{*}}\right)^{m} \\
& \times \exp \left\{-\frac{t}{w_{11} w_{22}-w_{12} w_{12}^{*}\left(1-\xi \xi^{*}\right)}\left[w_{11}+w_{22}+w_{12} \xi^{*}+w_{12}^{*} \xi\right]\right\} S(t) d t
\end{aligned}
$$

where $\mathbf{W}>\mathbf{0}$.

\footnotetext{
${ }^{5} a^{*}$ denotes the conjugate transpose of $a$.

${ }^{6} \Gamma_{2}(m)=\prod_{i=1}^{2} \pi \Gamma(m-i+1)=\pi \Gamma(m) \Gamma(m+1)$

${ }^{7} \operatorname{etr}(\cdot)$ defines $\exp (\operatorname{tr}(\cdot))$.
} 
Proof. Consider $\mathbf{W}=\mathbf{S}^{-1}$, with Jacobian $J=J\left(\mathbf{S} \rightarrow \mathbf{W}^{-1}\right)=|\mathbf{W}|^{-2 m}$ (see Maiwald and Kraus (1997)). From (3), it follows that the complex matrix variate inverse Wishart pdf is given by

$$
\begin{aligned}
f(\mathbf{W}) & =f_{\mathbf{S}}\left(\mathbf{W}^{-1}\right) J \\
& =\int_{\mathbb{R}^{+}} \frac{t^{2 m}}{\Gamma_{2}(m)|\mathbf{W}|^{m+2}|\mathbf{\Sigma}|^{m}} \operatorname{etr}\left(-t \boldsymbol{\Sigma}^{-1} \mathbf{W}^{-1}\right) S(t) d t
\end{aligned}
$$

where $\mathbf{W}>\mathbf{0}$, which leaves the final result.

Theorem 3 Suppose $\mathbf{W}$ follows a complex matrix variate inverse Wishart type distribution with pdf (5). Then the pdf of $\left(X_{1}, X_{2}\right)$, the inverse of the diagonals $W_{11}$ and $W_{22}$ of $\mathbf{W}$, is given by

$$
\begin{aligned}
f\left(x_{1}, x_{2}\right)= & \frac{2\left(1-a^{2}\right)}{\Gamma(m) \Gamma(m-1)} \sum_{k=0}^{\infty} \frac{1}{(k !)^{2}}\left(\frac{a^{2}}{1-a^{2}}\right)^{k}\left(x_{1} x_{2}\right)^{m+k-1} \\
& \times \sum_{p=0}^{k}\left(\begin{array}{l}
k \\
p
\end{array}\right)(-1)^{k+p} \frac{\left(1-a^{2}\right)^{p}}{\left(x_{1}+x_{2}\right)^{m+k+p+1}} \\
& \times \int_{\mathbb{R}^{+}} t^{m+k-p-1} \Gamma\left(m+k+p+1, \frac{t}{1-a^{2}}\left(x_{1}+x_{2}\right)\right) S(t) d t,
\end{aligned}
$$

where $x_{1}, x_{2}>0, m>0,0 \leq a^{2} \leq 1, \Gamma(\cdot)$ denotes the gamma function and $\Gamma(\cdot, \cdot)$ denotes the upper incomplete gamma function (see Gradshteyn and Ryzhik (2007), p. 899, eq. 8.350.2).

Proof. From (4), let

$$
\xi=a e^{-i b}, \quad a=|\xi|, \quad b=\arg (\xi) \text { and } 0 \leq a<1, w_{12}=\sqrt{w_{11} w_{22}} \rho e^{-i \theta}, \quad 0 \leq \theta<2 \pi, 0 \leq \rho<1,
$$

where $i^{2}=-1$ is the complex unitary, then $J=\left|2 \rho w_{11} w_{22}\right|$. It then follows that

$$
\begin{aligned}
f\left(w_{11}, w_{22}, \rho, \theta \mid t\right)= & \frac{t^{2 m}}{\Gamma_{2}(m)}\left(\frac{1}{w_{11} w_{22}\left(1-\rho^{2}\right)}\right)^{m+2}\left(\frac{1}{1-a^{2}}\right)^{m} \\
& \times \exp \left\{-\frac{t}{w_{11} w_{22}\left(1-\rho^{2}\right)\left(1-a^{2}\right)}\left[w_{11}+w_{22}+2 a \rho \sqrt{w_{11} w_{22}} \cos (\theta-b)\right]\right\} 2 \rho w_{11} w_{22} .
\end{aligned}
$$

The focus is to obtain the joint pdf of the inverted diagonal elements of $\mathbf{W}$. Making the transformations $X_{1}=W_{11}^{-1}$ and $X_{2}=W_{22}^{-1}$ with $J=\left(X_{1} X_{2}\right)^{-2}$ :

$$
\begin{aligned}
f\left(x_{1}, x_{2}, \rho, \theta \mid t\right)= & \frac{2 t^{2 m}\left(x_{1} x_{2}\right)^{m-1} \rho\left(1-\rho^{2}\right)^{-(m+2)}}{\pi\left(1-a^{2}\right)^{m} \Gamma(m) \Gamma(m-1)} \\
& \times \exp \left\{-\frac{t}{\left(1-\rho^{2}\right)\left(1-a^{2}\right)}\left[x_{1}+x_{2}+2 a \rho \sqrt{x_{1} x_{2}} \cos (\theta-b)\right]\right\} .
\end{aligned}
$$

The pdf of $\left(X_{1}, X_{2}, \rho, \theta\right)$ is given by

$$
\begin{aligned}
f\left(x_{1}, x_{2}, \rho, \theta\right)= & \int_{\mathbb{R}^{+}} f\left(x_{1}, x_{2}, \rho, \theta \mid t\right) S(t) d t \\
= & \frac{2\left(x_{1} x_{2}\right)^{m-1} \rho\left(1-\rho^{2}\right)^{-(m+2)}}{\pi\left(1-a^{2}\right)^{m} \Gamma(m) \Gamma(m-1)} \int_{\mathbb{R}^{+}} t^{2 m} S(t) \\
& \times \exp \left\{-\frac{t}{\left(1-\rho^{2}\right)\left(1-a^{2}\right)}\left[x_{1}+x_{2}+2 a \rho \sqrt{x_{1} x_{2}} \cos (\theta-b)\right]\right\} d t .
\end{aligned}
$$


Note that

$$
\begin{aligned}
I(A(t)) & =\int_{0}^{2 \pi} \exp (A(t) \cos (\theta-b)) d \theta \\
& =\sum_{k=0}^{\infty} \frac{2 \pi}{(k !)^{2}}\left(\frac{A(t)^{2}}{4}\right)^{k}
\end{aligned}
$$

where $A(t)=\frac{-2 t a \rho \sqrt{x_{1} x_{2}}}{\left(1-a^{2}\right)\left(1-\rho^{2}\right)}$ and $\int_{0}^{2 \pi} \cos ^{2 n+1} \theta d \theta=0 \quad \forall n$. Thus, we have that

$$
\begin{aligned}
f\left(x_{1}, x_{2}, \rho\right)= & \frac{4\left(x_{1} x_{2}\right)^{m-1} \rho\left(1-\rho^{2}\right)^{-(m+2)}}{\left(1-a^{2}\right)^{m} \Gamma(m) \Gamma(m-1)} \int_{\mathbb{R}^{+}} t^{2 m} S(t) \\
& \times \exp \left(-\frac{t\left(x_{1}+x_{2}\right)}{\left(1-\rho^{2}\right)\left(1-a^{2}\right)}\right) \sum_{k=0}^{\infty} \frac{1}{(k !)^{2}}\left(\frac{t^{2} a^{2} \rho^{2} x_{1} x_{2}}{\left(1-a^{2}\right)^{2}\left(1-\rho^{2}\right)^{2}}\right)^{k} d t .
\end{aligned}
$$

To obtain the pdf of $\left(X_{1}, X_{2}\right)$, let $Z_{i}=X_{i} /\left(1-a^{2}\right), i=1,2$, and $Y=\frac{1}{\left(1-\rho^{2}\right)}$, with $J=\left(1-a^{2}\right)^{2}$. Then the pdf of the bivariate gamma type is given by

$$
f\left(x_{1}, x_{2}\right)=\left(\frac{1}{1-a^{2}}\right)^{2} f_{Z_{1}, Z_{2}}\left(\frac{x_{1}}{1-a^{2}}, \frac{x_{2}}{1-a^{2}}\right)
$$

with pdf of $\left(Z_{1}, Z_{2}, Y\right)$ given by

$$
\begin{aligned}
f\left(z_{1}, z_{2}, y\right)= & \frac{2\left(1-a^{2}\right)^{m}}{\Gamma(m) \Gamma(m-1)} \sum_{k=0}^{\infty} \frac{a^{2 k}}{(k !)^{2}}\left(z_{1} z_{2}\right)^{m+k-1} y^{m+k}(y-1)^{k} \\
& \times \int_{\mathbb{R}^{+}} t^{2(m+k)} \exp \left\{-t\left(z_{1}+z_{2}\right) y\right\} S(t) d t .
\end{aligned}
$$

Subsequently, the pdf of $\left(Z_{1}, Z_{2}\right)$ has form

$$
\begin{aligned}
f\left(z_{1}, z_{2}\right)= & \int_{1}^{\infty} f\left(z_{1}, z_{2}, y\right) d y \\
= & \int_{1}^{\infty} \frac{2\left(1-a^{2}\right)^{m}}{\Gamma(m) \Gamma(m-1)} \sum_{k=0}^{\infty} \frac{a^{2 k}}{(k !)^{2}}\left(z_{1} z_{2}\right)^{m+k-1} y^{m+k}(y-1)^{k} \\
& \times \int_{\mathbb{R}^{+}} t^{2(m+k)} \exp \left\{-t\left(z_{1}+z_{2}\right) y\right\} S(t) d t d y \\
= & \frac{2\left(1-a^{2}\right)^{m}}{\Gamma(m) \Gamma(m-1)} \sum_{k=0}^{\infty} \frac{a^{2 k}}{(k !)^{2}}\left(z_{1} z_{2}\right)^{m+k-1} \sum_{p=0}^{k}\left(\begin{array}{c}
k \\
p
\end{array}\right) \frac{(-1)^{k+p}}{\left(z_{1}+z_{2}\right)^{m+k+p+1}} \\
& \times \int_{\mathbb{R}^{+}} t^{m+k-p-1} \Gamma\left(m+k+p+1, t\left(z_{1}+z_{2}\right)\right) S(t) d t,
\end{aligned}
$$

using Gradshteyn and Ryzhik (2007), p. 346, eq. 3.381.3. Therefore from (9) and (11), $\left(X_{1}, X_{2}\right)$ has the 
following bivariate gamma type pdf

$$
\begin{aligned}
f\left(x_{1}, x_{2}\right)= & \frac{2\left(1-a^{2}\right)}{\Gamma(m) \Gamma(m-1)} \sum_{k=0}^{\infty} \frac{1}{(k !)^{2}}\left(\frac{a^{2}}{1-a^{2}}\right)^{k}\left(x_{1} x_{2}\right)^{m+k-1} \\
& \times \sum_{p=0}^{k}\left(\begin{array}{l}
k \\
p
\end{array}\right)(-1)^{k+p} \frac{\left(1-a^{2}\right)^{p}}{\left(x_{1}+x_{2}\right)^{m+k+p+1}} \\
& \times \int_{\mathbb{R}^{+}} t^{m+k-p-1} \Gamma\left(m+k+p+1, \frac{t}{1-a^{2}}\left(x_{1}+x_{2}\right)\right) S(t) d t,
\end{aligned}
$$

where $x_{1}, x_{2}>0$, leaving the final result.

\subsection{Graphical representation}

In this section the graphs of pdf for the bivariate gamma type distribution (6) are displayed for two special cases, namely the complex matrix variate normal and the complex matrix variate $t$ distributions as underlying models for $\mathbf{H}$.

Corollary 4 For the bivariate gamma distribution, let (see Provost and Cheong (2002))

$$
S(t)=\delta(t-1)
$$

where $\delta(\cdot)$ is the dirac delta or impulse function having the property $\int_{\mathbb{R}^{+}} f(x) \delta(x) d x=f(0)$, for every Borel-measurable function $f(\cdot)$, i.e. $\mathbf{H}$ has a complex matrix variate normal distribution. We then obtain the result of Xu et. al. (2009). Specifically, let $x=t-1$, then

$$
\begin{aligned}
I_{1} & =\int_{\mathbb{R}^{+}} t^{m+k-p-1} \Gamma\left(m+k+p+1, \frac{t}{1-a^{2}}\left(x_{1}+x_{2}\right)\right) \delta(t-1) d t \\
& =\Gamma\left(m+k+p+1, \frac{1}{1-a^{2}}\left(x_{1}+x_{2}\right)\right)
\end{aligned}
$$

which simplifies to the bivariate gamma distribution

$$
\begin{aligned}
f_{\text {normal }}\left(x_{1}, x_{2}\right)= & \frac{2\left(1-a^{2}\right)}{\Gamma(m) \Gamma(m-1)} \sum_{k=0}^{\infty} \frac{1}{(k !)^{2}}\left(\frac{a^{2}}{1-a^{2}}\right)^{k}\left(x_{1} x_{2}\right)^{m+k-1} \\
& \times \sum_{p=0}^{k}\left(\begin{array}{l}
k \\
p
\end{array}\right) \frac{(-1)^{k+p}\left(1-a^{2}\right)^{p}}{\left(x_{1}+x_{2}\right)^{m+k+p+1}} \Gamma\left(m+k+p+1, \frac{1}{1-a^{2}}\left(x_{1}+x_{2}\right)\right),
\end{aligned}
$$

where $x_{1}, x_{2}>0$.

Corollary $\mathbf{5}$ For the bivariate gamma $t$ distribution, let

$$
S(t)=\frac{\left(\frac{v}{2}\right)^{\frac{v}{2}}}{\Gamma\left(\frac{\nu}{2}\right)} t^{\frac{v}{2}-1} e^{-\frac{v t}{2}}
$$


i.e. $\mathbf{H}$ has a complex matrix variate $t$ distribution with $v>0$ degrees of freedom. Using Gradshteyn and Ryzhik (2007), p. 65\%, eq. 6.455.1, observe that:

$$
\begin{aligned}
I_{2}= & \frac{\left(\frac{\nu}{2}\right)^{\frac{\nu}{2}}}{\Gamma\left(\frac{\nu}{2}\right)} \int_{\mathbb{R}^{+}} t^{\frac{\nu}{2}+m+k-p-2} e^{-\frac{\nu t}{2}} \Gamma\left(m+k+p+1, \frac{t}{1-a^{2}}\left(x_{1}+x_{2}\right)\right) d t \\
= & \frac{\left(\frac{\nu}{2}\right)^{\frac{\nu}{2}}}{\Gamma\left(\frac{\nu}{2}\right)} \frac{\left(\frac{x_{1}+x_{2}}{1-a^{2}}\right)^{m+k+p+1} \Gamma\left(\frac{\nu}{2}+2 m+2 k\right)}{\left(\frac{\nu}{2}+m+k-p-1\right)\left(\frac{x_{1}+x_{2}}{1-a^{2}}+\frac{\nu}{2}\right)^{\frac{\nu}{2}+2 m+2 k}} \\
& \times{ }_{2} F_{1}\left(1, \frac{\nu}{2}+2 m+2 k ; \frac{\nu}{2}+m+k-p ; \frac{\frac{\nu}{2}}{\frac{x_{1}+x_{2}}{1-a^{2}}+\frac{\nu}{2}}\right)
\end{aligned}
$$

where ${ }_{2} F_{1}(\cdot)$ denotes the Gauss hypergeometric function (see Gradshteyn and Ryzhik (2007), p. 1010, eq. 9.14.2). The final expression for the pdf of bivariate gamma $t$ distribution with $v$ degrees of freedom has form

$$
\begin{aligned}
f_{t}\left(x_{1}, x_{2}\right)= & \frac{2\left(\frac{\nu}{2}\right)^{\frac{\nu}{2}}}{\Gamma(m) \Gamma(m-1) \Gamma\left(\frac{\nu}{2}\right)\left(1-a^{2}\right)^{m-2 k}} \sum_{k=0}^{\infty} \frac{a^{2 k} \Gamma\left(\frac{\nu}{2}+2 m+2 k\right)}{(k !)^{2}} \\
& \times\left(x_{1} x_{2}\right)^{m+k-1} \sum_{p=0}^{k}\left(\begin{array}{l}
k \\
p
\end{array}\right)(-1)^{k+p} \\
& \times \frac{{ }_{2} F_{1}\left(1, \frac{\nu}{2}+2 m+2 k ; \frac{\nu}{2}+m+k-p ; \frac{\frac{\nu}{2}}{\frac{x_{1}+x_{2}}{1-a^{2}}+\frac{\nu}{2}}\right)}{\left(\frac{\nu}{2}+m+k-p-1\right)\left(\frac{x_{1}+x_{2}}{1-a^{2}}+\frac{\nu}{2}\right)^{\frac{\nu}{2}+2 m+2 k}},
\end{aligned}
$$

where $x_{1}, x_{2}>0$.

In Figure 1 through 4 the nature of pdfs $(13)$ and $(15)$ is illustrated for some arbitrary parameters.
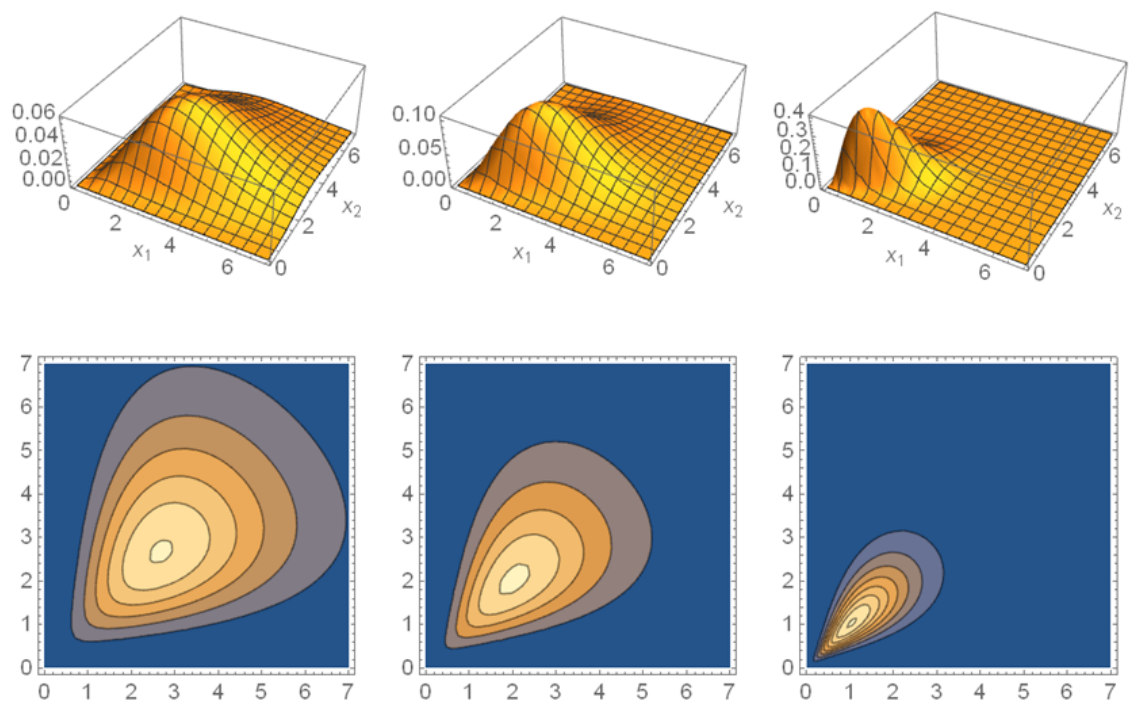

Figure 1 Pdf 13 with contourplots for $m=5$ and $a=0.25,0.5,0.75$ (fltr) 

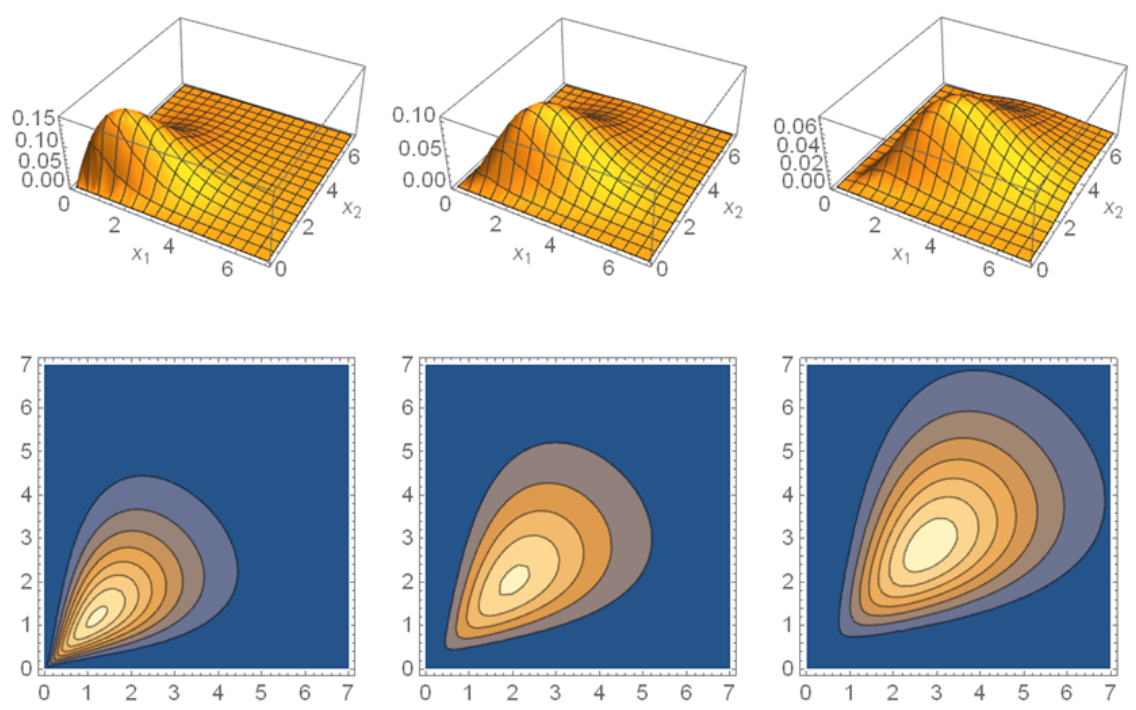

Figure 2 Pdf 13 with contourplots for $a=0.5, v=10$ and $m=4,5,6$ (fltr)
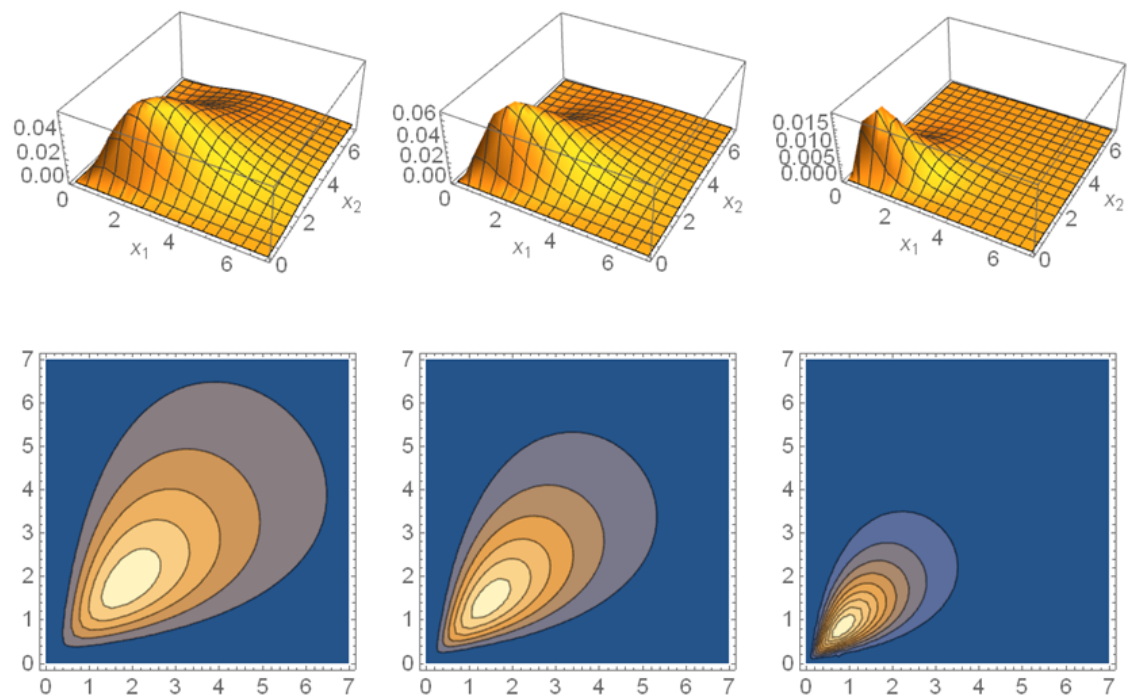

Figure 3 Pdf (15) with contourplots for $m=5, v=10$ and $a=0.25,0.5,0.75$ (fltr) 

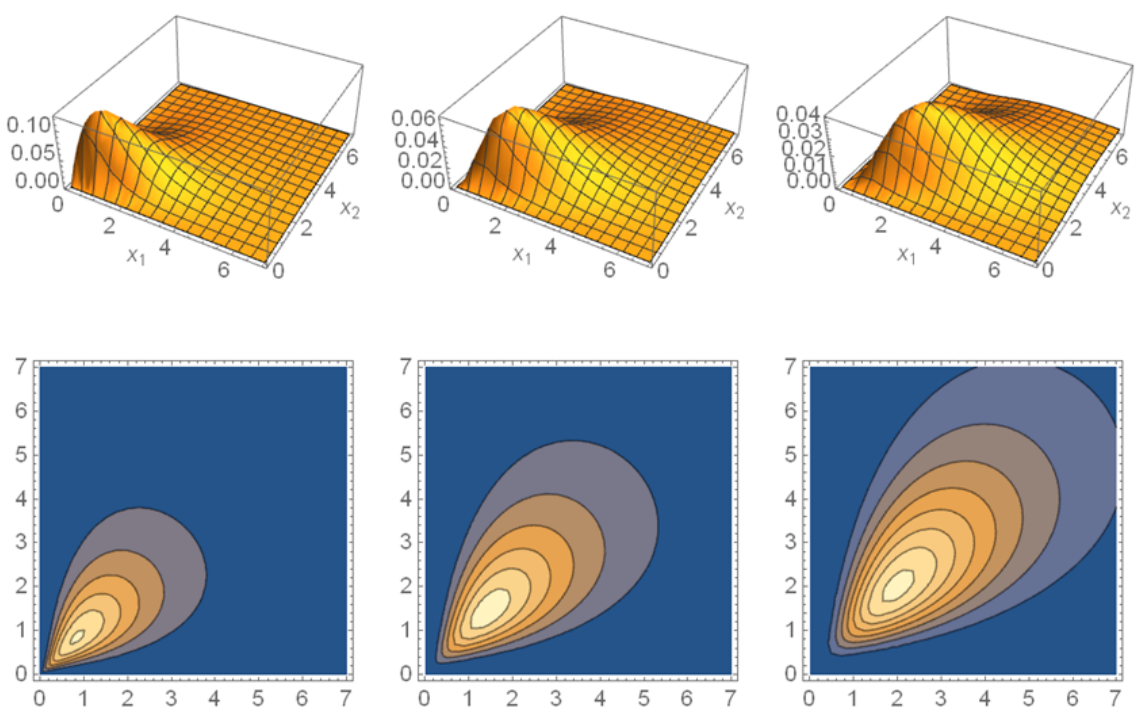

Figure $4 \mathrm{Pdf} 15$ with contourplots for $a=0.5, v=10$ and $m=4,5,6$ (fltr)

It is observed from Figures 1 and 3 that when $a$ approaches 1, the pdf becomes more dense. The underlying $t$ case, in comparison to the normal distribution, exhibits fatter tails as expected.

\subsection{Product moments}

In this section, the product moments of the bivariate gamma type distribution with pdf (6) is derived. Subsequently the expression of the product moments is obtained for both underlying complex matrix variate normal- and $t$ cases, and the Pearson correlation coefficient investigated.

Remark 6 The product moment of $\left(X_{1}, X_{2}\right)$ with pdf (6) is given by

$$
E\left(X_{1}^{r} X_{2}^{n}\right)=\left(1-a^{2}\right)^{r+n} E\left(Z_{1}^{r} Z_{2}^{n}\right)
$$

with

$$
\begin{aligned}
E\left(Z_{1}^{r} Z_{2}^{n}\right)= & \int_{\mathbb{R}^{+}} \int_{\mathbb{R}^{+}} \int_{1}^{\infty} z_{1}^{r} z_{2}^{n} f\left(z_{1}, z_{2}, y\right) d z_{1} d z_{2} d y \\
= & \int_{\mathbb{R}^{+}} \int_{\mathbb{R}^{+}} \int_{1}^{\infty} z_{1}^{r} z_{2}^{n} \frac{2\left(1-a^{2}\right)^{m}}{\Gamma(m) \Gamma(m-1)} \sum_{k=0}^{\infty} \frac{a^{2 k}}{(k !)^{2}}\left(z_{1} z_{2}\right)^{m+k-1} y^{m+k}(y-1)^{k} \\
& \times \int_{\mathbb{R}^{+}} t^{2(m+k)} \exp \left\{-t y\left(z_{1}+z_{2}\right)\right\} S(t) d t d z_{1} d z_{2} d y \\
= & \frac{2\left(1-a^{2}\right)^{m}}{\Gamma(m) \Gamma(m-1)} \sum_{k=0}^{\infty} \frac{a^{2 k}}{(k !)^{2}} \int_{1}^{\infty} \int_{\mathbb{R}^{+}} t^{-r-m-k-n-m-k+2 m+2 k} \\
& \times \frac{\Gamma(r+m+k)}{\Gamma(n+m+k)} \frac{\Gamma\left(1-a^{2}\right)^{m}}{y^{n+m+k}} y^{m+k}(y-1)^{k} S(t) d t d y \\
= & \frac{a^{2 k}}{\Gamma(m) \Gamma(m-1)} \sum_{k=0}^{\infty} \frac{(k !)^{2}}{(r+m+k) \Gamma(n+m+k)} \\
& \times \int_{\mathbb{R}^{+}} t^{-(r+n)} S(t) d t \int_{1}^{\infty} y^{-(r+n+m+k)}(y-1)^{k} d y
\end{aligned}
$$


using (10) and Gradshteyn and Ryzhik (2007), p. 346, eq. 3.381.4. Further using Gradshteyn and Ryzhik (2007), p. 315, eq. 3.191.2:

$$
\begin{aligned}
E\left(Z_{1}^{r} Z_{2}^{n}\right)= & \frac{2\left(1-a^{2}\right)^{m}}{\Gamma(m) \Gamma(m-1)} \sum_{k=0}^{\infty} \frac{a^{2 k}}{(k !)^{2}} \Gamma(r+m+k) \Gamma(n+m+k) \\
& \times \int_{\mathbb{R}^{+}} t^{-(r+n)} S(t) d t B(r+n+m-1, k+1) \\
= & \frac{2 \kappa(r+n)\left(1-a^{2}\right)^{m}}{\Gamma(m) \Gamma(m-1)} \sum_{k=0}^{\infty} \frac{a^{2 k}}{(k !)^{2}} \Gamma(r+m+k) \Gamma(n+m+k) \\
& \times \frac{\Gamma(r+n+m-1) \Gamma(k+1)}{\Gamma(r+n+m+k)} \\
= & \frac{2 \kappa(r+n)\left(1-a^{2}\right)^{m}}{\Gamma(m) \Gamma(m-1)} \frac{\Gamma(m+r) \Gamma(m+n)}{(m+r+n-1)}{ }_{2} F_{1}\left(r, n ; r+n+m ; a^{2}\right)
\end{aligned}
$$

using the definition of the Pochhammer symbol, and where $B(\cdot, \cdot)$ is the beta function, and (provided it exists)

$$
\kappa(u)=\int_{\mathbb{R}^{+}} t^{-u} S(t) d t .
$$

Corollary 7 The product moment of $\left(X_{1}, X_{2}\right)$ with pdf (13) is given by

$$
E_{\text {normal }}\left(X_{1}^{r} X_{2}^{n}\right)=\frac{2\left(1-a^{2}\right)^{r+n+m}}{\Gamma(m) \Gamma(m-1)} \frac{\Gamma(m+r) \Gamma(m+n)}{(m+r+n-1)}{ }_{2} F_{1}\left(r, n ; r+n+m ; a^{2}\right)
$$

using (12).

Corollary 8 The product moment of $\left(X_{1}, X_{2}\right)$ with pdf 15$)$ is given by

$$
\begin{aligned}
E_{t}\left(X_{1}^{r} X_{2}^{n}\right)= & \frac{2\left(1-a^{2}\right)^{r+n+m}\left(\frac{v}{2}\right)^{r+n} \Gamma\left(\frac{v}{2}-(r+n)\right) \Gamma(m+r) \Gamma(m+n)}{\Gamma(m) \Gamma(m-1) \Gamma\left(\frac{\nu}{2}\right)(m+r+n-1)} \\
& \times{ }_{2} F_{1}\left(r, n ; r+n+m ; a^{2}\right)
\end{aligned}
$$

using (14) and Gradshteyn and Ryzhik (2007), p. 346, eq. 3.381.4.

By using the expressions for the product moment in $(19)$ and $(20)$, the Pearson correlation coefficient can now be determined using $\rho_{X_{1}, X_{2}}=\frac{E\left(X_{1} X_{2}\right)-E\left(X_{1}\right) E\left(X_{2}\right)}{\sqrt{E\left(X_{1}^{2}\right)-E\left(X_{1}\right)^{2}} \sqrt{E\left(X_{2}^{2}\right)-E\left(X_{2}\right)^{2}}}$. In Figure 5 this correlation coefficient is graphed for arbitrary parameter values $(m=3, v=5,15)$ against $a^{2}$ to illustrate the effect of $a^{2}$ on the correlation between $X_{1}$ and $X_{2}$. 


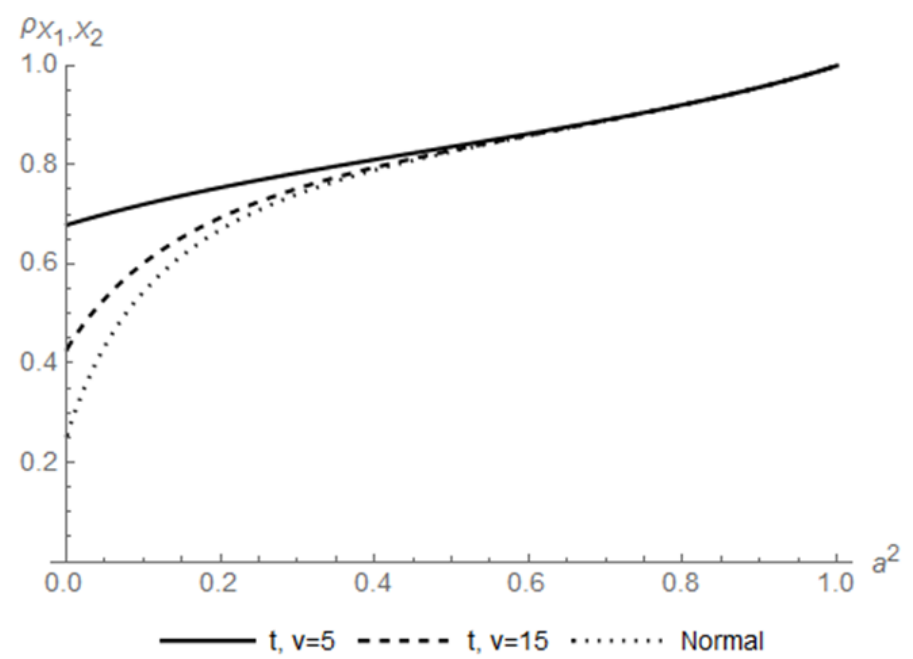

Figure 5 Pearson's correlation coefficient for increasing values of $a^{2}$

Corollary 9 The moment generating function (mgf) of $\left(X_{1}, X_{2}\right)$ is given by

$$
M\left(q_{1}, q_{2}\right)=\sum_{j=0}^{\infty} \frac{1}{j !} \sum_{i=0}^{j}\left(\begin{array}{l}
j \\
i
\end{array}\right) q_{1}^{i} q_{2}^{j-i} \frac{2 \kappa(j)\left(1-a^{2}\right)^{m+j}}{\Gamma(m) \Gamma(m-1)} \frac{\Gamma(m+i) \Gamma(m+j-i)}{(m+j-1)}{ }_{2} F_{1}\left(i, j-i ; j+m ; a^{2}\right)
$$

where $m>0,0<a^{2}<1$, and with $\kappa(j)$ as defined in (18).

Proof. Substituting (16) and (17) into the definition of the mgf, together with Gradshteyn and Ryzhik (2007), p. 25, eq. 1.111, yield

$$
\begin{aligned}
M\left(q_{1}, q_{2}\right)= & E\left(\sum_{j=0}^{\infty} \frac{1}{j !}\left(q_{1} X_{1}+q_{2} X_{2}\right)^{j}\right) \\
= & \sum_{j=0}^{\infty} \frac{1}{j !} \sum_{i=0}^{j}\left(\begin{array}{l}
j \\
i
\end{array}\right) q_{1}^{i} q_{2}^{j-i} E\left(X_{1}^{i} X_{2}^{j-i}\right) \\
= & \sum_{j=0}^{\infty} \frac{1}{j !} \sum_{i=0}^{j}\left(\begin{array}{l}
j \\
i
\end{array}\right) q_{1}^{i} q_{2}^{j-i}\left(1-a^{2}\right)^{i+(j-i)} E_{Z_{1}, Z_{2}}\left(Z_{1}^{i} Z_{2}^{j-i}\right) \\
= & \sum_{j=0}^{\infty} \frac{1}{j !} \sum_{i=0}^{j}\left(\begin{array}{l}
j \\
i
\end{array}\right) q_{1}^{i} q_{2}^{j-i}\left(1-a^{2}\right)^{j} \frac{2 \kappa(j)\left(1-a^{2}\right)^{m}}{\Gamma(m) \Gamma(m-1)} \\
& \times \frac{\Gamma(m+i) \Gamma(m+j-i)}{(m+j-1)}{ }_{2} F_{1}\left(i, j-i ; j+m ; a^{2}\right) \\
= & \sum_{j=0}^{\infty} \frac{1}{j !} \sum_{i=0}^{j}\left(\begin{array}{l}
j \\
i
\end{array}\right) q_{1}^{i} q_{2}^{j-i} \frac{2 \kappa(j)\left(1-a^{2}\right)^{m+j}}{\Gamma(m) \Gamma(m-1)} \\
& \times \frac{\Gamma(m+i) \Gamma(m+j-i)}{(m+j-1)}{ }_{2} F_{1}\left(i, j-i ; j+m ; a^{2}\right)
\end{aligned}
$$

which concludes the proof. 


\subsection{Bivariate Weibullised gamma type distribution}

Bivariate Nakagami- $m$ distribution has many applications in wireless communications (see de Souza and Yacoub (2008) and references therein). A bivariate Nakagami- $m$ type distribution that emanates from this bivariate gamma type distribution is thus of particular interest. A bivariate Weibullised gamma type distribution (see also Chen et. al. (2014)) is proposed, which originates from the bivariate gamma type distribution (see (6)), of which the bivariate Nakagami- $m$ type distribution is a special case.

Theorem 10 Suppose that $\left(X_{1}, X_{2}\right)$ is bivariate gamma type distributed with pdf (6). The pdf of $\left(R_{1}, R_{2}\right)$, where $R_{i}=\left(\frac{X_{i}}{m}\right)^{\frac{1}{\beta_{i}}}$ is given by

$$
\begin{aligned}
f\left(r_{1}, r_{2}\right)= & \frac{2 \beta_{1} \beta_{2}}{\Gamma(m) \Gamma(m-1)} \sum_{k=0}^{\infty} \sum_{p=0}^{k} \frac{\left(\begin{array}{l}
k \\
p
\end{array}\right)(-1)^{k+p}\left(1-a^{2}\right)^{p+1}}{(k !)^{2}} m^{m+k-p-1}\left(\frac{a^{2}}{1-a^{2}}\right)^{k} \frac{r_{1}^{\beta_{1} m+\beta_{1} k-1} r_{2}^{\beta_{2} m+k \beta_{2}-1}}{\left(r_{1}^{\beta_{1}}+r_{2}^{\beta_{2}}\right)^{m+k+p+1}} \\
& \times \int_{\mathbb{R}^{+}} t^{m+k-p-1} \Gamma\left(m+k+p+1, \frac{t}{1-a^{2}}\left(m r_{1}^{\beta_{1}}+m r_{2}^{\beta_{2}}\right)\right) S(t) d t
\end{aligned}
$$

for $r_{1}, r_{2}>0, m, \beta_{1}, \beta_{2}>0$ and $0<a^{2}<1$. This distribution is called a bivariate Weibullised gamma type distribution.

Proof. Consider the transformations $R_{1}=\left(\frac{X_{1}}{m}\right)^{\frac{1}{\beta_{1}}}$ and $R_{2}=\left(\frac{X_{2}}{m}\right)^{\frac{1}{\beta_{2}}}$ with $J=\beta_{1} \beta_{2} m^{2} r_{1}^{\beta_{1}-1} r_{2}^{\beta_{2}-1}$. The pdf of $\left(R_{1}, R_{2}\right)$ is obtained from (6):

$$
\begin{aligned}
f\left(r_{1}, r_{2}\right)= & \frac{2\left(1-a^{2}\right)}{\Gamma(m) \Gamma(m-1)} \sum_{k=0}^{\infty} \frac{1}{(k !)^{2}}\left(\frac{a^{2}}{1-a^{2}}\right)^{k}\left(m r_{1}^{\beta_{1}} m r_{2}^{\beta_{2}}\right)^{m+k-1} \\
& \times \sum_{p=0}^{k}\left(\begin{array}{l}
k \\
p
\end{array}\right)(-1)^{k+p} \frac{\left(1-a^{2}\right)^{p}}{\left(m r_{1}^{\beta_{1}}+m r_{2}^{\beta_{2}}\right)^{m+k+p+1}} \\
& \times \int_{\mathbb{R}^{+}} t^{m+k-p-1} \Gamma\left(m+k+p+1, \frac{t}{1-a^{2}}\left(m r_{1}^{\beta_{1}}+m r_{2}^{\beta_{2}}\right)\right) S(t) d t \beta_{1} \beta_{2} m^{2} r_{1}^{\beta_{1}-1} r_{2}^{\beta_{2}-1} \\
= & \frac{2 \beta_{1} \beta_{2}}{\Gamma(m) \Gamma(m-1)} \sum_{k=0}^{\infty} \frac{1}{(k !)^{2}}\left(\frac{a^{2}}{1-a^{2}}\right)^{k} r_{1}^{\beta_{1} m+\beta_{1} k-1} r_{2}^{\beta_{2} m+k \beta_{2}-1} \sum_{p=0}^{k}\left(\begin{array}{l}
k \\
p
\end{array}\right)(-1)^{k+p} m^{m+k-p-1} \\
& \times \frac{\left(1-a^{2}\right)^{p+1}}{\left(r_{1}^{\beta_{1}}+r_{2}^{\beta_{2}}\right)^{m+k+p+1}} \int_{\mathbb{R}^{+}} t^{m+k-p-1} \Gamma\left(m+k+p+1, \frac{t}{1-a^{2}}\left(m r_{1}^{\beta_{1}}+m r_{2}^{\beta_{2}}\right)\right) S(t) d t
\end{aligned}
$$

which concludes the result.

Remark 11 When $\beta_{1}=\beta_{2}=2$, then (21) has the pdf of a bivariate Nakagami-m type distribution:

$$
\begin{aligned}
f\left(r_{1}, r_{2}\right)= & \frac{4\left(1-a^{2}\right) m^{2 m}}{\Gamma(m) \Gamma(m-1)} \sum_{k=0}^{\infty} \sum_{p=0}^{k} \frac{\left(\begin{array}{l}
k \\
p
\end{array}\right)(-1)^{k+p}}{(k !)^{2}}\left(\frac{a^{2} m^{2}}{1-a^{2}}\right)^{k}\left(r_{1} r_{2}\right)^{2 m+2 k-1} \frac{\left(1-a^{2}\right)^{p}}{\left(m r_{1}^{2}+m r_{2}^{2}\right)^{m+k+p+1}} \\
& \times \int_{\mathbb{R}^{+}} t^{m+k-p-1} \Gamma\left(m+k+p+1, \frac{t}{1-a^{2}}\left(m r_{1}^{2}+m r_{2}^{2}\right)\right) S(t) d t,
\end{aligned}
$$

for $r_{1}, r_{2}>0, m>0,0<a^{2}<1$. For the special cases under consideration, observe the following: 
i) Substituting (12) into (20), a bivariate Nakagami-m distribution has pdf

$$
\begin{aligned}
f_{\text {normal }}\left(r_{1}, r_{2}\right)= & \frac{4\left(1-a^{2}\right) m^{2 m}}{\Gamma(m) \Gamma(m-1)} \sum_{k=0}^{\infty} \sum_{p=0}^{k} \frac{\left(\begin{array}{l}
k \\
p
\end{array}\right)(-1)^{k+p}}{(k !)^{2}}\left(\frac{a^{2} m^{2}}{1-a^{2}}\right)^{k}\left(r_{1} r_{2}\right)^{2 m+2 k-1} \\
& \times \frac{\left(1-a^{2}\right)^{p}}{\left(m r_{1}^{2}+m r_{2}^{2}\right)^{m+k+p+1}} \Gamma\left(m+k+p+1, \frac{1}{1-a^{2}}\left(m r_{1}^{2}+m r_{2}^{2}\right)\right),
\end{aligned}
$$

where $r_{1}, r_{2}>0$.

ii) Substituting (14) into (22), a bivariate Nakagami-m $t$ distribution has pdf

$$
\begin{aligned}
f_{t}\left(r_{1}, r_{2}\right)= & \frac{4\left(1-a^{2}\right) m^{2 m}\left(\frac{v}{2}\right)^{\frac{v}{2}}}{\Gamma(m) \Gamma(m-1) \Gamma\left(\frac{v}{2}\right)} \sum_{k=0}^{\infty} \sum_{p=0}^{k} \frac{\left(\begin{array}{l}
k \\
p
\end{array}\right)(-1)^{k+p}}{(k !)^{2}}\left(\frac{a^{2} m^{2}}{1-a^{2}}\right)^{k} \frac{\left(1-a^{2}\right)^{p}\left(r_{1} r_{2}\right)^{2 m+2 k-1}}{\left(m r_{1}^{2}+m r_{2}^{2}\right)^{m+k+p+1}} \\
& \times \int_{\mathbb{R}^{+}} t^{m+k+\frac{v}{2}-p-2} e^{-\frac{v}{2} t} \Gamma\left(m+k+p+1, \frac{t}{1-a^{2}}\left(m r_{1}^{2}+m r_{2}^{2}\right)\right) d t,
\end{aligned}
$$

where $r_{1,} r_{2}>0$.

In Figures 6 and 7 the nature of pdfs $(23)$ and $(24)$ is illustrated for some arbitrary parameters.
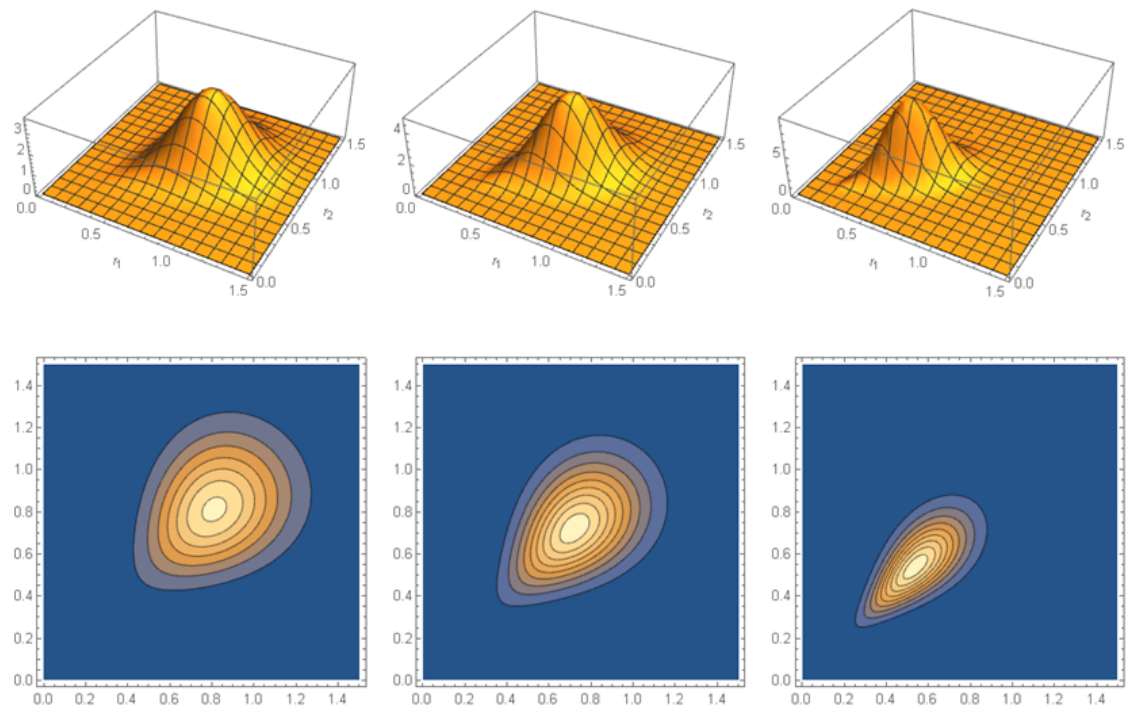

Figure 6 Pdf (23) with contourplots for $m=5$ and $a=0.25,0.5,0.75$ (fltr) 

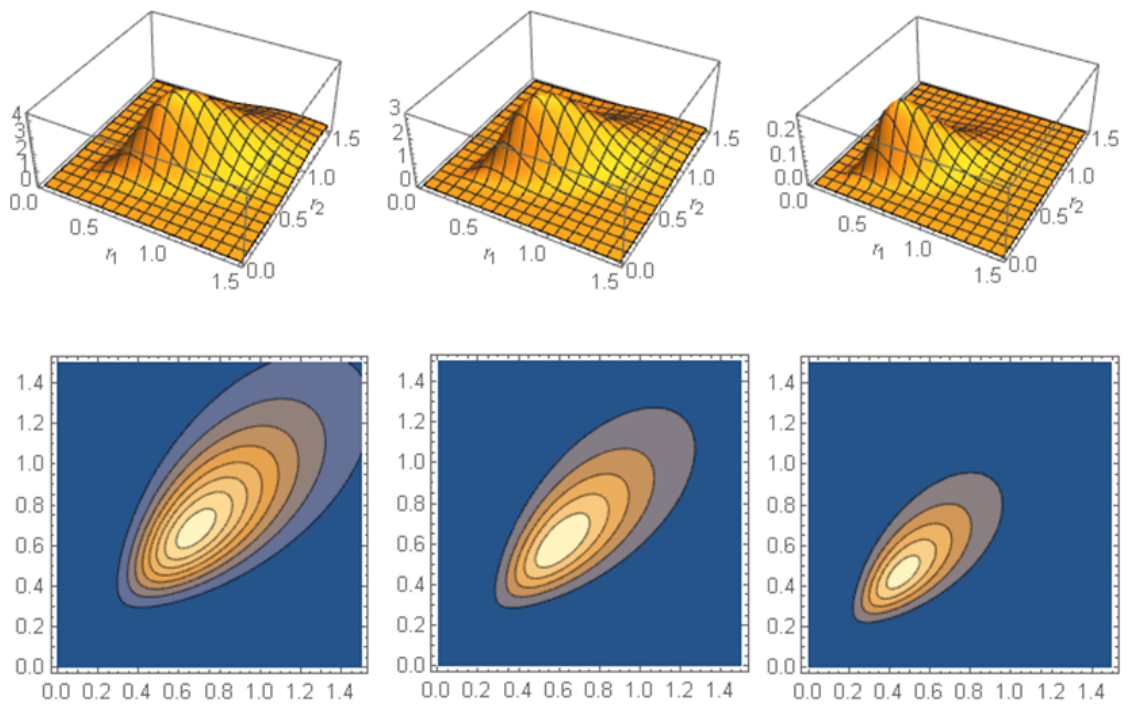

Figure 7 Pdf 24 ) with contourplots for $m=5, v=5$ and $a=0.25,0.5,0.75$ (fltr)

As before, it is observed from Figure 6 that when $a$ approaches one, the pdf becomes more dense.

\section{MIMO Applications}

In this section, possible applications are proposed of the newly developed models in section 2 .

\subsection{Application in Rayleigh type fading environment}

Let $\mathbf{H}$ be the matrix for MIMO system with two transmit antennas, where $\mathbf{H} \sim C E_{m, 2}\left(\mathbf{0}, \mathbf{I}_{m} \otimes \boldsymbol{\Sigma}, h\right)$ and is subject to Rayleigh type fading (see Proposition 1). Based on our notation, the outage probability at a certain threshold $x_{t h}$ is obtained as (see Simon and Alouini (2005))

$$
\hat{F}\left(x_{t h}\right)=F\left(\frac{x_{t h}}{1-a^{2}}\right)
$$

where

$$
F(z)=P\left\{\max \left(Z_{1}, Z_{2}\right)<z\right\} .
$$

Thus, we need to compute $F(\cdot)$, the cumulative distribution function (cdf) of $\max \left(Z_{1}, Z_{2}\right)$. To this end, using (10) and Gradshteyn and Ryzhik (2007), p. 346, eq. 3.381.1 we obtain

$$
\begin{aligned}
P\left(z_{1}<z, z_{2}<z, y\right) & =\int_{0}^{z} \int_{0}^{z} f\left(z_{1}, z_{2}, y\right) d z_{1} d z_{2} \\
& =\frac{2\left(1-a^{2}\right)^{m}}{\Gamma(m) \Gamma(m-1)} \sum_{k=0}^{\infty} \frac{a^{2 k}}{(k !)^{2}} y^{-(m+k)}(y-1)^{k} \int_{\mathbb{R}^{+}} \gamma^{2}(m+k ; t y z) S(t) d t
\end{aligned}
$$


where $\gamma(\cdot, \cdot)$ denotes the lower incomplete gamma function (see Gradshteyn and Ryzhik (2007), p. 899, eq. 8.350.1). Subsequently

$$
\begin{aligned}
F(z) & =\int_{1}^{\infty} P\left(z_{1}<z, z_{2}<z, y\right) d y \\
& =\frac{2\left(1-a^{2}\right)^{m}}{\Gamma(m) \Gamma(m-1)} \sum_{k=0}^{\infty} \frac{a^{2 k}}{(k !)^{2}} \int_{1}^{\infty} \int_{\mathbb{R}^{+}} y^{-(m+k)}(y-1)^{k} \gamma^{2}(m+k ; t y z) S(t) d t d y .
\end{aligned}
$$

Finally, for the underlying complex matrix variate normal- and $t$ cases (see $(12)$ and (14)), the cdf is given by

$$
F_{\text {normal }}(z)=\frac{2\left(1-a^{2}\right)^{m}}{\Gamma(m) \Gamma(m-1)} \sum_{k=0}^{\infty} \frac{a^{2 k}}{(k !)^{2}} \int_{1}^{\infty} y^{-(m+k)}(y-1)^{k} \gamma^{2}(m+k ; y z) d y
$$

and

$$
F_{t}(z)=\frac{2\left(1-a^{2}\right)^{m}\left(\frac{v}{2}\right)^{\frac{v}{2}}}{\Gamma(m) \Gamma(m-1) \Gamma\left(\frac{v}{2}\right)} \sum_{k=0}^{\infty} \frac{a^{2 k}}{(k !)^{2}} \int_{1}^{\infty} y^{-(m+k)}(y-1)^{k} \int_{\mathbb{R}^{+}} \gamma^{2}(m+k ; t y z) t^{\frac{v}{2}-1} e^{-\frac{v}{2} t} d t d y
$$

respectively. These expressions are evaluated numerically next.

Remark 12 Different expressions and methods are available (see Gradshteyn and Ryzhik (2007) p. 347, eq. 3.383.3 and p. 899, eq. 8.351.2) for simplified expressions (26) and (27). However, due to cumbersome computational execution, the authors utilize a direct integration of these expressions.

A simulation is conducted using Matlab to validate the analytical results. The results and accompanying figures are illustrated using Mathematica. Figure 8 illustrates the outage probability of the signal-to-noise ratio (SNR) for MIMO systems with 2 transmitters and $m=3$ receivers over correlated Rayleigh type channels with underlying complex matrix variate $t$ distribution. The analytical results (solid line) match the simulated results (dashed line) closely, which can be observed by Table 1 indicating selected values of the outage probability for the analytical- and simulation studies.

Figures $9 \mathrm{a}$ and $9 \mathrm{~b}$ illustrate the results for the underlying complex matrix variate $t$ distribution together with underlying complex matrix variate normal distribution also for the case of 2 transmitters and 3 receivers for $a=0.5$. The different behaviour for small and large outage thresholds is noteworthy, as evident from Figures 9a and 9b. This observation provides significant insight to the theoretical contribution of the candidacy of the complex matrix variate $t$ distribution in comparison to the complex matrix variate normal case. Note that Figure $9 \mathrm{~b}$ is a magnified version of Figure 9a over a subset of the domain. 


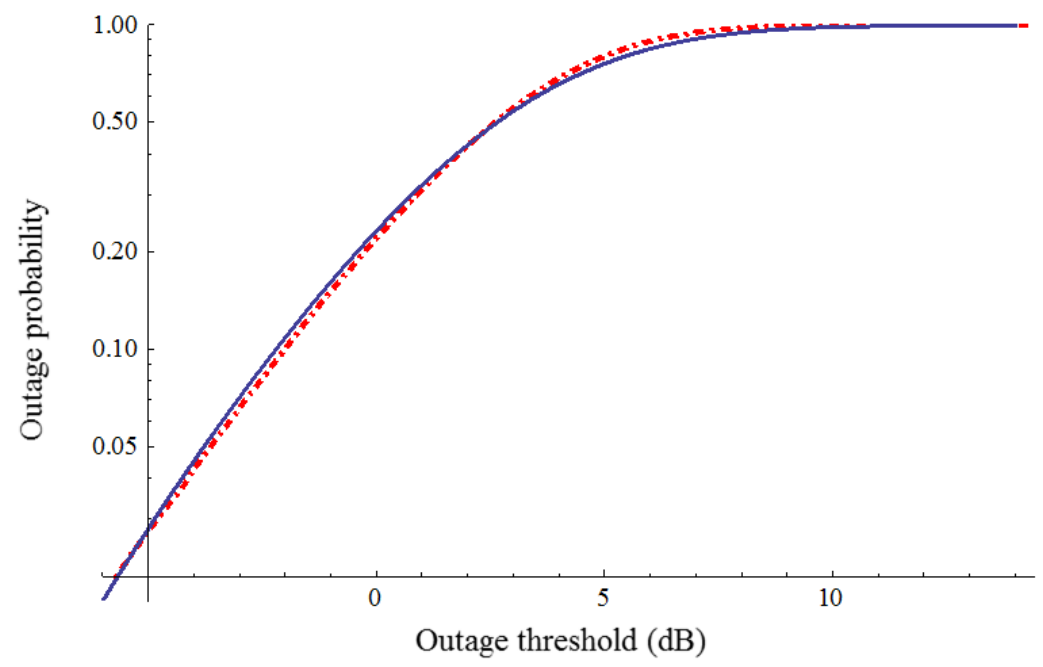

Figure 8 Analytical (27) and simulated outage probabilities, for $a=0.5, v=10$

\begin{tabular}{|l||l|l|}
\hline Outage threshold & Analytical & Simulation \\
\hline \hline-4 & 0.0453594 & 0.04279 \\
\hline-2 & 0.108779 & 0.10027 \\
\hline 0 & 0.231966 & 0.22034 \\
\hline 2 & 0.426804 & 0.42380 \\
\hline 4 & 0.656033 & 0.68335 \\
\hline 6 & 0.84272 & 0.88991 \\
\hline 8 & 0.944624 & 0.97745 \\
\hline 10 & 0.98230 & 0.99725 \\
\hline
\end{tabular}

Table 1 Analytical (27) and simulated outage probabilities, for $a=0.5, v=10$ 

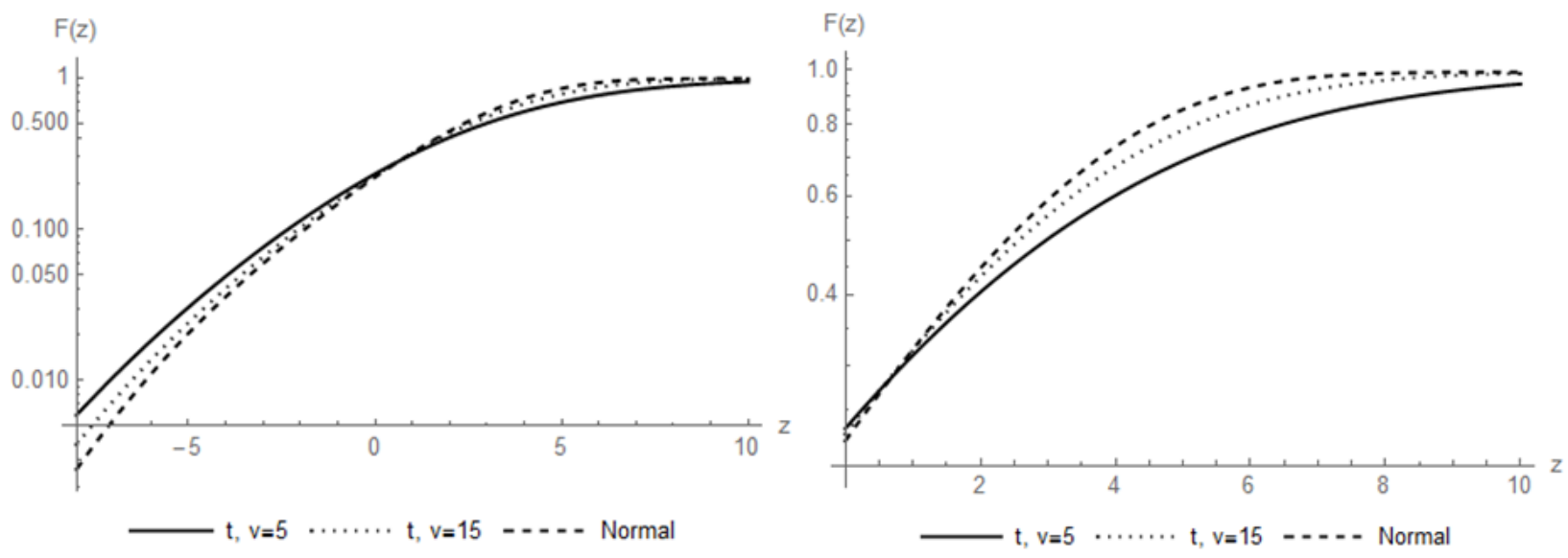

Figure 9a (left) and 9b (right) Analytical outage probabilities 27 against output threshold, for $a=0.5$

Table 2 gives further insight by providing the value of the outage probability versus the outage threshold, corresponding to Figure 9a. The values in bold indicate when the outage probability is lowest between the three candidate fittings.

\begin{tabular}{|l||l|l|l|}
\hline Outage threshold & $\begin{array}{l}\text { Outage probability } \\
(t, v=5)\end{array}$ & $\begin{array}{l}\text { Outage probability } \\
(t, v=15)\end{array}$ & $\begin{array}{l}\text { Outage probability } \\
(\text { normal })\end{array}$ \\
\hline \hline-2 & 0.11684 & 0.105532 & $\mathbf{0 . 0 9 8 0 3 4 8}$ \\
\hline-1.5 & 0.140931 & 0.129676 & $\mathbf{0 . 1 2 1 6 7 7}$ \\
\hline-1 & 0.168608 & 0.158206 & $\mathbf{0 . 1 5 0 0 9 5}$ \\
\hline-0.5 & 0.199999 & 0.191543 & $\mathbf{0 . 1 8 3 9 5 7}$ \\
\hline 0 & 0.235115 & 0.230001 & $\mathbf{0 . 2 2 3 8 7 7}$ \\
\hline 0.5 & 0.273826 & 0.273717 & $\mathbf{0 . 2 7 0 3 3 3}$ \\
\hline 1 & $\mathbf{0 . 3 1 5 8 4 3}$ & $\mathbf{0 . 3 2 2 5 9}$ & 0.323553 \\
\hline 1.5 & $\mathbf{0 . 3 6 0 7 1 9}$ & $\mathbf{0 . 3 7 6 2 1 5}$ & 0.383382 \\
\hline 2 & $\mathbf{0 . 4 0 7 8 5 1}$ & $\mathbf{0 . 4 3 3 8 4 4}$ & 0.44914 \\
\hline 2.5 & $\mathbf{0 . 4 5 6 5 1 4}$ & $\mathbf{0 . 4 9 4 3 7 9}$ & 0.519513 \\
\hline 3 & $\mathbf{0 . 5 0 5 8 9 4}$ & $\mathbf{0 . 5 5 6 4 1 1}$ & 0.592509 \\
\hline
\end{tabular}

Table 2 Analytical outage probabilities against the output threshold, for $a=0.5$

\subsection{Equal gain diversity}

Based on the proposed bivariate Nakagami- $m$ type fading distribution with pdf (22), the performance of a 2-branch predetection equal gain combiner (EGC) over the bivariate Nakagami- $m$ type fading channels is investigated (see Karagiannidis (2004)). To this end, an expression is determined for the moments of the EGC output signal-to-noise ratio (SNR) assuming a bivariate Nakagami- $m$ type fading channel (see (22). 
Now, suppose that in a fading environment, a 2-branch predetection EGC diversity receiver is operating. Then, the instantaneous output SNR per symbol (see Simon and Alouini (2005)) is given by

$$
\gamma_{\text {out }}=\frac{E_{s}}{2 N_{o}}\left(R_{1}+R_{2}\right)^{2}
$$

where $E_{s}$ is energy per symbol. Hence, the $n^{\text {th }}$ moment of the EGC output SNR is evaluated using (11), (18), Gradshteyn and Ryzhik (2007), p. 315, eq. 3.191.2, and Gradshteyn and Ryzhik (2007), p. 25, eq. 1.111 as

$$
\mu_{n}=E\left(\gamma_{\text {out }}\right)^{n}=\left(\frac{E_{s}}{2 N_{o}}\right)^{n} \sum_{s=0}^{2 n}\left(\begin{array}{c}
2 n \\
s
\end{array}\right) \int_{\mathbb{R}^{+}} \int_{\mathbb{R}^{+}} r_{1}^{s} r_{2}^{2 n-s} f\left(r_{1}, r_{2}\right) d r_{1} d r_{2}
$$

It follows that

$$
\begin{aligned}
\mu_{n}= & 4 m^{2}\left(\frac{1}{1-a^{2}}\right)^{2}\left(\frac{E_{s}}{2 N_{o}}\right)^{n} \sum_{s=0}^{2 n}\left(\begin{array}{c}
2 n \\
s
\end{array}\right) \frac{2\left(1-a^{2}\right)^{m}}{\Gamma(m) \Gamma(m-1)} \sum_{k=0}^{\infty} \frac{a^{2 k}}{(k !)^{2}} \\
& \times \int_{\mathbb{R}^{+}} \int_{\mathbb{R}^{+}} r_{1}^{s} r_{2}^{2 n-s}\left(\frac{\theta_{1} r_{1}^{2}}{\left(1-a^{2}\right)} \times \frac{\theta_{2} r_{2}^{2}}{\left(1-a^{2}\right)}\right)^{m+k-1} \int_{1}^{\infty} y^{r+k}(y-1)^{k} \\
& \times \int_{\mathbb{R}^{+}} t^{2(m+k)} \exp \left(-t y\left(\frac{\theta_{1} r_{1}^{2}}{\left(1-a^{2}\right)}+\frac{\theta_{2} r_{2}^{2}}{\left(1-a^{2}\right)}\right)\right) S(t) r_{1} r_{2} d t d y d r_{1} d r_{2} \\
= & B(2 n ; s, k) \int_{\mathbb{R}^{+}} t^{2(m+k)}\left(\int_{y=1}^{\infty} I_{3}(t, y) y^{m+k}(y-1)^{k} d y\right) S(t) d t
\end{aligned}
$$

where

$$
B(2 n ; s, k)=4 m^{2}\left(\frac{1}{1-a^{2}}\right)^{2}\left(\frac{E_{s}}{2 N_{o}}\right)^{n} \sum_{s=0}^{2 n}\left(\begin{array}{c}
2 n \\
s
\end{array}\right) \frac{2\left(1-a^{2}\right)^{r}}{\Gamma(r) \Gamma(r-1)} \sum_{k=0}^{\infty} \frac{a^{2 k}}{(k !)^{2}}
$$

and

$$
\begin{aligned}
I_{3}(t, y)= & \int_{\mathbb{R}^{+}} \int_{\mathbb{R}^{+}} r_{1}^{s+1} r_{2}^{2 n-s+1}\left(\frac{m r_{1}^{2}}{\left(1-a^{2}\right)} \times \frac{m r_{2}^{2}}{\left(1-a^{2}\right)}\right)^{m+k-1} \\
& \times \exp \left(-t y\left(\frac{m r_{1}^{2}}{\left(1-a^{2}\right)}+\frac{m r_{2}^{2}}{\left(1-a^{2}\right)}\right)\right) d r_{1} d r_{2} \\
= & \frac{1}{4}\left(1-a^{2}\right)^{n+2}\left(\frac{1}{m}\right)^{n-\frac{s}{2}+1}\left(\frac{1}{m}\right)^{\frac{s}{2}+1} \frac{1}{(t y)^{n+2 m+2 k-1}} \\
& \times \Gamma\left(n-\frac{s}{2}+m+k\right) \Gamma\left(\frac{s}{2}+m+k\right)
\end{aligned}
$$

using Gradshteyn and Ryzhik (2007), p. 346, eq. 3.381.4. Consequently, using (18) and 
Gradshteyn and Ryzhik (2007), p. 315, eq. 3.191.2 we obtain

$$
\begin{aligned}
\mu_{n}= & \frac{1}{4} B(2 n)\left(1-a^{2}\right)^{n+2}\left(\frac{1}{m}\right)^{n-\frac{s}{2}+1}\left(\frac{1}{m}\right)^{\frac{s}{2}+1} \\
& \times \Gamma\left(n-\frac{s}{2}+m+k\right) \Gamma\left(\frac{s}{2}+m+k\right) \\
& \times \int_{\mathbb{R}^{+}} t^{2(m+k)}\left(\int_{1}^{\infty} \frac{y^{m+k}(y-1)^{k}}{(t y)^{n+m+k}} d y\right) S(t) d t \\
= & \frac{1}{4} B(2 n)\left(1-a^{2}\right)^{n+2}\left(\frac{1}{m}\right)^{n-\frac{s}{2}+1}\left(\frac{1}{m}\right)^{\frac{s}{2}+1} \kappa(n) \\
& \times \Gamma\left(n-\frac{s}{2}+m+k\right) \Gamma\left(\frac{s}{2}+m+k\right) B(n+m-1, k+1)
\end{aligned}
$$

After some algebra a final expression for the EGD (assuming a bivariate Nakagami- $m$ type distribution) is given by

$$
\begin{aligned}
\mu_{n}= & \frac{2\left(1-a^{2}\right)^{m+n} \kappa(n)}{\Gamma(m) \Gamma(m-1)}\left(\frac{E_{s}}{2 N_{o}}\right)^{n} \sum_{s=0}^{2 n}\left(\begin{array}{c}
2 n \\
s
\end{array}\right) \sum_{k=0}^{\infty} \frac{a^{2 k}}{(k !)^{2}}\left(\frac{1}{m}\right)^{n-\frac{s}{2}}\left(\frac{1}{m}\right)^{\frac{s}{2}} \\
& \times \Gamma\left(n-\frac{s}{2}+m+k\right) \Gamma\left(\frac{s}{2}+m+k\right) B(n+m-1, k+1) .
\end{aligned}
$$

The expressions for EGD for the normal- and $t$ case respectively are given by

$$
\begin{aligned}
\mu_{n, \text { normal }}= & \frac{2\left(1-a^{2}\right)^{m+n}}{\Gamma(m) \Gamma(m-1) m^{n}}\left(\frac{E_{s}}{2 N_{o}}\right)^{n} \sum_{s=0}^{2 n}\left(\begin{array}{c}
2 n \\
s
\end{array}\right) \sum_{k=0}^{\infty} \frac{a^{2 k}}{(k !)^{2}} \\
& \times \Gamma\left(n-\frac{s}{2}+m+k\right) \Gamma\left(\frac{s}{2}+m+k\right) B(n+m-1, k+1)
\end{aligned}
$$

and

$$
\begin{aligned}
\mu_{n, t}= & \frac{2\left(1-a^{2}\right)^{m+n}\left(\frac{v}{2}\right)^{n} \Gamma\left(\frac{v}{2}-n\right)}{\Gamma(m) \Gamma(m-1) \Gamma\left(\frac{v}{2}\right) m^{n}}\left(\frac{E_{s}}{2 N_{o}}\right)^{n} \sum_{s=0}^{2 n}\left(\begin{array}{c}
2 n \\
s
\end{array}\right) \sum_{k=0}^{\infty} \frac{a^{2 k}}{(k !)^{2}} \\
& \times \Gamma\left(n-\frac{s}{2}+m+k\right) \Gamma\left(\frac{s}{2}+m+k\right) B(n+m-1, k+1) .
\end{aligned}
$$

In Figures 10 and 11 the behaviour of the EGD $(\sqrt{28})$ and $(29))$ is illustrated for some arbitrary parameters. 


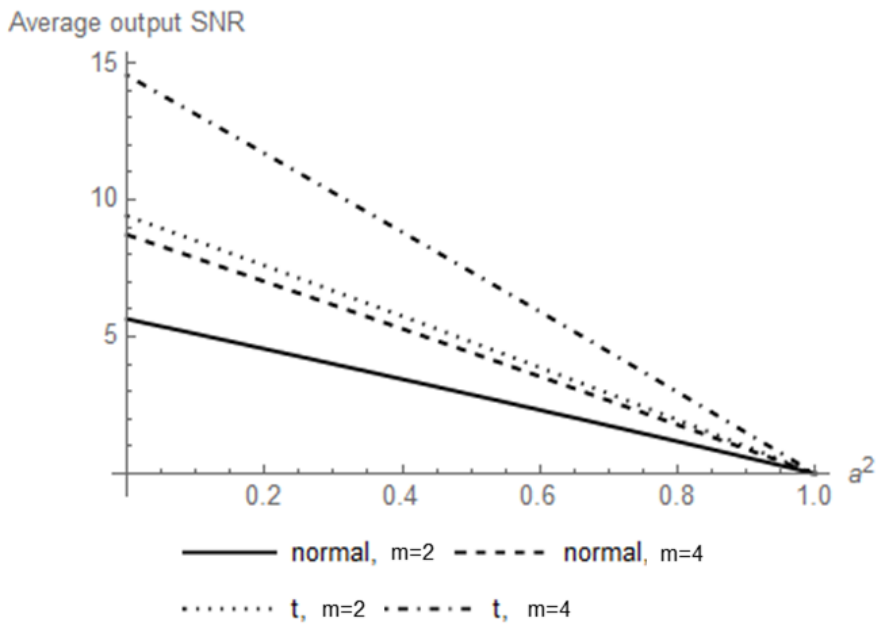

Figure $10 \sqrt{28}$ and $(29)$ against $a$, for different values of $m, v=5, n=1$

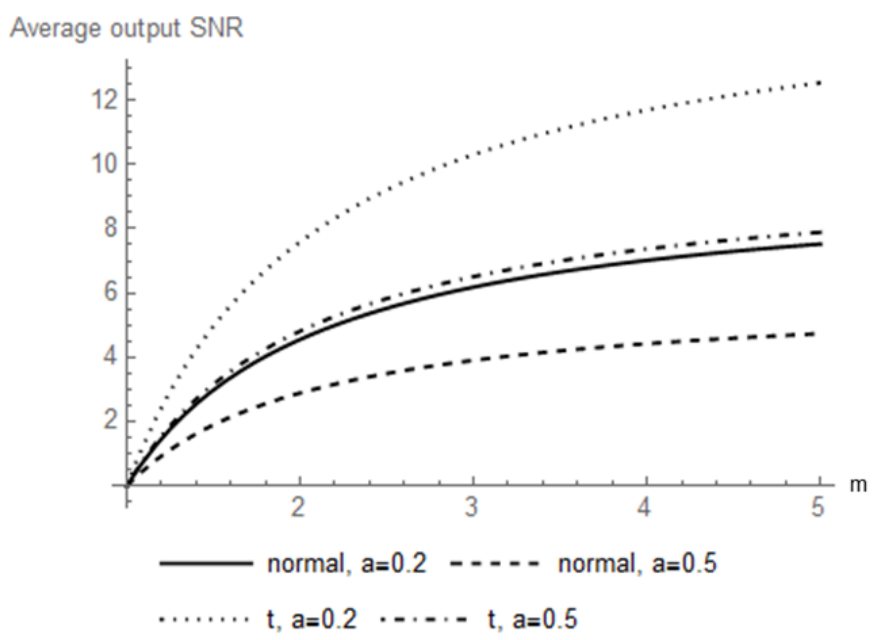

Figure $11(28)$ and $(29)$ against $m$, for different values of $a, v=5, n=1$

It is observed that correlation between transmitters decreases the EGD severely. Even so, for the underlying $t$ distribution, the EGD is higher than that of the normal case. Furthermore for both cases, as the number of receivers increase the EGD increases correspondingly.

\section{Conclusion}

In this paper new bivariate gamma- and bivariate Weibullised gamma type distributions have been presented which originated from the diagonal elements of a complex inverse Wishart type distribution. Specifically, the pdf, cdf, and product moments of the bivariate gamma type distribution have been derived. Since the complex elliptical class constitutes a flexible- and broad class of distributions, this paper provides some insight in the possible usefulness for engineering applications by this assumption. The results have been applied to evaluate the outage probability of a MIMO system with two transmit antennas with underlying models the complex matrix variate normal- and $t$ case. In future, different 
members of the complex elliptical class may be explored as possible candidates versus the well-studiedand assumed normal model in this MIMO field (Clavier (2017)).

\section{Acknowledgements}

The authors would like to thank the anonymous referee for the constructive comments which improved the presentation of the paper. The authors acknowledge the support of the StatDisT group. This work is based upon research supported by the National Research Foundation, South Africa (ref. CPRR13090132066 grant nr 91497 \& ref. CPRR160403161466 grant nr. 105840). M. Arashi's research is supported in part by the National Research Foundation of South Africa (grant nr. 109214).

\section{References}

[Balakrishnan and Lai (2002)]

[Clavier (2017)]

[de Souza and Yacoub (2008)]

[de Souza et. al. (2012)]
[Chen et. al. (2014)]

Balakrishnan, N. and Lai, C. (2002). Bivariate probability distributions, $2^{\text {nd }}$ Edition. Springer-Verlag, New York.

Chen, L., Tzeng, I.S., and Lin, C. (2014). Bivariate generalized gamma distributions of Kibble's type, Statistics, 48(4), 933 - 949.

Clavier, L. (2017). Modeling interference with alpha-stable distributions and copulae for receiver design in wireless communications, Contributed session, 61st ISI World Congress in Statistics, Marrakech, Morocco.

de Souza, R.A.A. and Yacoub, M.D. (2008). Bivariate Nakagami-m distribution with arbitrary correlation and fading parameters, IEEE Transactions on Wireless Communications, 7(12), 5227-5232.

de Souza, R.A.A., Yacoub, M.D., and Rabelo, G.S. (2012). Bivariate Hoyt (Nakagami-q) distribution, IEEE Transactions in Communications, 6(12), 714-721.

[Ermelova and Tirkonnen (2015)] Ermelova, N.Y. and Tirkkonen, O. (2015). Cumulative distribution function of bivariate gamma distribution with arbitrary parameters and applications, IEEE Communications Letters, 19(2), 1-4.

[Gradshteyn and Ryzhik (2007)] Gradshteyn, I.S. and Ryzhik, I.M. (2007). Table of Integral, Series, and Products, 7th Ed., Academic Press, Oxford, UK.

[Hoyt (1947)]

Hoyt, R.S. (1947). Probability functions for the modulus and angle of the normal complex variate, Bell System Technical Journal, 26, 318359.

[Karagiannidis (2004)]

Karagiannidis, G.K. (2004). Moments-based approach to the performance analysis of equal gain diversity in Nakagami-m fading, IEEE Transactions on Communications, 52(5), 685-689.

Mahdavi, A., Fathizadeh, M., and Jamalizadeh, A. (2017) On the bivariate weighted exponential distribution based on the generalized exponential distribution, Communications in Statistics - Theory and Methods, 46(8), 3641-3648. 
[Maiwald and Kraus (1997)]

[Miller (1974)]

[Nakagami (1960)]

[Ollila et. al. (2011)]

[Provost and Cheong (2002)]

[Simon and Alouini (2005)]

[Telatar (1999)]

[Xu et. al. (2009)]
Maiwald, D. and Kraus, D. (1997). On moments of complex Wishart and complex inverse Wishart distributed matrices, IEEE International Conference on Acoustics, Speech, and Signal Processing Conference Proceedings, 5, 3817-3820.

Miller, K.S. (1974). Complex stochastic processes: an introduction to theory and application, Addison-Wesley, New York.

Nakagami, M. (1960). The $m$-distribution - a general formula of intensity distribution of rapid fading, Statistical methods in radio wave propagation (Ed: Hoffman W.G.). Pergamon.

Ollila, E., Eriksson, J., and Koivunen, V. (2011). Complex elliptically symmetric random variables - generation, characterisation, and circularity tests, IEEE Transactions on Signal Processing, 59(1), 58-69.

Provost, S.B. and Cheong, Y.-Ho. (2002). The distribution of Hermitian quadratic forms in elliptically contoured random vectors, Journal of Statistical Planning and Inference, 102, 303-316.

Simon, M. and Alouini, M. (2005). Digital communication over fading channels. John Wiley and Sons, New York.

Telatar, I.E. (1999). Capacity of multi-antenna Gaussian channels, Europian Transactions in Telecommunications, 10, 585-595.

Xu, R., Zhong, Z., Chen, J-M., and Ai, B. (2009). Bivariate gamma distribution from complex inverse Wishart matrix, IEEE Communications Letters, 13(2), 118-120. 